\title{
Fingerprint Deformation Models Using Minutiae Locations and Orientations
}

\author{
Yi Chen, Sarat Dass, Arun Ross, and Anil Jain \\ Department of Computer Science and Engineering \\ Michigan State University \\ East Lansing, MI, 48823
}

\begin{abstract}
Nonlinear deformations in fingerprint images, arising from the elasticity of the skin as well as the pressure and movement of the finger during image acquisition, lead to difficulties in establishing a match between multiple impressions acquired from the same finger. One solution to this problem is to estimate and remove the relative deformations prior to the matching stage. In this paper, these relative deformations are represented as an average deformation model based on minutiae locations and orientations using 2-D Thin Plate Splines (TPS). The estimated average deformation is used to pre-distort a template prior to matching it with a query image in a verification task. Experimental results show that the use of minutiae locations and orientations to estimate the deformation leads to a more representative deformation model than using minutiae locations only. An index of deformation based on the bending energy is also proposed to select templates with the least variability in the deformations. The EER goes down by $\approx 1.1 \%$ when we incorporate minutiae orientation information and use the template selection strategy.
\end{abstract}

\section{Introduction}

There are several factors that impact fingerprint deformation: the amount of pressure applied by the subject, the disposition of the subject (sitting or standing), the motion of the finger, the moisture content of the skin (dry, oily or wet), the elasticity of the skin. Recently, several methods have been presented to deal with this deformation from different viewpoints. Ratha et al. [3] proposed a controlled acquisition process by measuring the forces and torques on the scanner directly with the aid of specialized hardware. Senior et al. [4] suggested an automatic method to remove deformations by enforcing the constraint that ridges should be constantly spaced, with deviations from constant spacings indicating the presence of deformations. Watson et al. [12] constructed deformation filters for template fingerprint images prior to using a correlation-based matching. KovácsVajna [7] proposed a matching method that takes deforma-
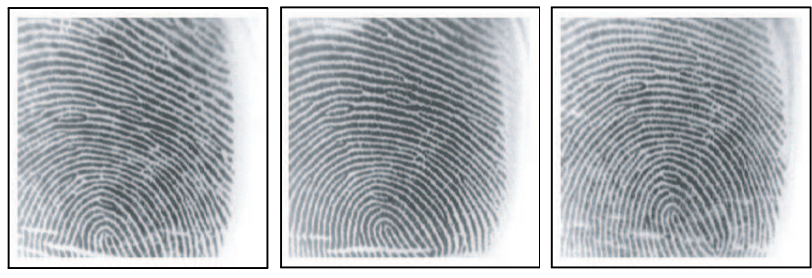

Figure 1: Three impressions of a single fi nger showing the effect of varying deformations.

tions into account by employing tolerance bounds for interminutiae distances and angles. Bazen et al. [5,6] used a Thin-Plate Spline (TPS) model to describe the non-linear deformations between two minutiae sets. However, most of these techniques deal with the problem of non-linear deformation on a case-by-case basis. No attempt was made to develop a finger-specific deformation model that can be computed offline and then used for matching. The main advantage of an offline technique is that once a finger-specific model has been computed, recomputation of the model is not necessary during the matching stage. Also, by using multiple impressions of the same finger to compute deformations from a template, we avoid estimating optimal deformations for impostor pairs, resulting in low FAR values.

In [1], we have proposed such a finger-specific deformation model using 2-D TPS based on minutiae point patterns. The TPS, a spatial generalization of the cubic spline, is an effective tool for estimating the deformation that warps one set of point patterns to another. The TPS model in [1] is computed by distorting the minutiae set of one fingerprint impression to match with others from the same finger. Although the deformations observed in a fingerprint vary from one acquisition to the next (Fig.1), it forms an informative and representative feature space which is best characterized through statistical modeling. However, this method only employed the locations of minutiae points during deformation estimation, ignoring the fact that orientations of minutiae points also provide additional and useful information for a better estimate of the deformation.

In this paper, both the orientation and location information of minutiae are incorporated in the TPS model. A new 
method to establish minutiae correspondences is also developed to compensate for the sensitivity of the TPS model to incorrect correspondences. Incorrect correspondences are caused due to false, missed or displaced minutiae points, or when there is limited overlap between image pairs, resulting in an unreliable TPS model. We also introduce the use of the variance of the "bending energy," instead of pixel-wise covariance matrix of deformations as in [1], to rank the relative deformations associated with a finger. We demonstrate that using the variance of the "bending energy" leads to greater computational efficiency and a better performance compared to [1].

The rest of the paper is organized as follows. Section 2 describes the general methodology, including the basic concepts of the TPS model. Section 3 gives a modified algorithm for obtaining reliable correspondences. Section 4 describes the transformations needed for incorporating the minutiae orientations in the TPS model. Section 5 develops an average deformation model for a template and ranks templates of the same finger based on the variance of the "bending energy." Experimental results are provided in Section 6, with summary and future work presented in Section 7.

\section{General Methodology}

Given a pair of grayscale fingerprint images, $I_{0}$ and $I_{1}$, defined on a spatial domain $R^{2}$, the deformation from $I_{0}$ to $I_{1}$ is given by a deformation function $F: R^{2} \rightarrow R^{2}$ such that $F\left(I_{0}\right)=I_{1}$. In this paper, we focus on modelling $F$ using the Thin-Plate Splines (TPS). Pioneered by Bookstein [8], the TPS modelling is particularly useful when two or threedimensional landmarks in a reference need to be mapped to the corresponding landmarks in a target such that the "bending energy" of $F$ is minimized.

Specifically, let $U=\left(u_{1}, u_{2}, \ldots, u_{l}\right)^{T}$ and $V=\left(v_{1}, v_{2}\right.$, $\left.\ldots, u_{l}\right)^{T}$ be a pair of point sets with known correspondences, derived from $I_{0}$ and $I_{1}$, respectively. We assume $U$ and $V$ have been aligned using the Procrustes analysis [10]. Here, $u_{k}$ and $v_{k}$ denote the spatial coordinates of the $k$ th corresponding pair and $l$ is the total number of correspondences. The deformation function $F$ is then required to satisfy

$$
F\left(u_{i}\right)=v_{i}, \quad i=1,2, \ldots, l .
$$

The TPS estimate of $F$ is given by parameter vectors $c, A$ and $W$ :

$$
F(u)=c+A \cdot u+W^{T} s(u),
$$

where $u \in R^{2}, c$ and $A$ define the affine parts of the transformation and $W$ gives the additional non-linear deformation. The distance measure $s(u)$ is the vector $(\sigma(u-$ $\left.\left.u_{1}\right), \sigma\left(u-u_{2}\right), \ldots, \sigma\left(u-u_{l}\right)\right)^{T}$ with

$$
\sigma(u)=\|u\|^{2} \log \|u\| .
$$

A total of $(6+2 l)$ parameters in equation (2) need to be estimated, where $c$ is a $2 \times 1$ vector, $A$ is a $2 \times 2$ matrix and $W$ is a $l \times 2$ matrix. Since equation (1) enforces $l$ constraints in the spatial domain, we can reduce the number of degrees of freedom by $2 l$. Further, we assume that the coefficients $W$ satisfy (i) $1_{l}^{T} W=0$ (2 restrictions) and (ii) $U^{T} W=0$ (4 restrictions), where $1_{l}$ is a vector of ones of length $l$. Thus, the coefficients of the TPS model can be obtained from the matrix equation:

$$
\left[\begin{array}{ccc}
S & 1_{l} & U \\
1_{l}^{T} & 0 & 0 \\
U^{T} & 0 & 0
\end{array}\right]\left[\begin{array}{c}
W \\
c^{T} \\
A^{T}
\end{array}\right]=\left[\begin{array}{l}
V \\
0 \\
0
\end{array}\right]
$$

where $S$ is the $l \times l$ matrix of $\left(\sigma\left(u_{j}-u_{k}\right)\right), j, k=1,2, \ldots, l$. The above matrix gives rise to a TPS model that minimizes the bending energy subject to the perfect alignment constraints in equation (1). A more robust TPS model can be obtained by relaxing the constraints in equation (1) and redefining the transformation $F$ which minimizes the expression

$$
\sum_{j=1}^{l}\left(v_{j}-F\left(u_{j}\right)\right)^{T}\left(v_{i}-F\left(u_{i}\right)\right)+\lambda J(F),
$$

where

$$
\begin{aligned}
J(F)= & \sum_{j=1}^{2} \int_{R^{2}}\left\{\left(\frac{\partial^{2} F_{j}(x, y)}{\partial x^{2}}\right)^{2}+2\left(\frac{\partial^{2} F_{j}(x, y)}{\partial x \partial y}\right)^{2}\right. \\
& \left.+\left(\frac{\partial^{2} F_{j}(x, y)}{\partial y^{2}}\right)^{2}\right\} d x d y
\end{aligned}
$$

represents the bending energy associated with $F=$ $\left(F_{1}, F_{2}\right)^{T}$, with $F_{j}$ as the $j$-th component of $F$, and $\lambda>0$. The coefficients of this resulting TPS model can be obtained using equation (4) with $S$ replaced by $S+\lambda I_{l}$, where $I_{l}$ is the $l \times l$ identity matrix. Generally, these splines do not exactly interpolate all landmark points, but are allowed to approximate them in favor of a smoothing parameter $\lambda$. When $\lambda$ increases, the resulting spline becomes more smooth.

\section{Establishing Point Correspondences}

The TPS model relies on correct correspondences of point patterns during deformation estimation. Thus, for fingerprint images, the accuracy of finding minutiae correspondences can greatly affect the efficacy and efficiency of the TPS. In order to obtain reliable minutiae correspondences, we modified the Elastic Point Pattern Matching (EPPM) algorithm proposed in [9] by incorporating a voting scheme to find the optimal global translation and rotation. The procedure is described as follows: 


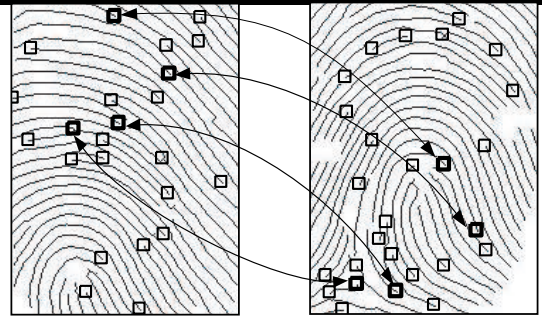

(a)

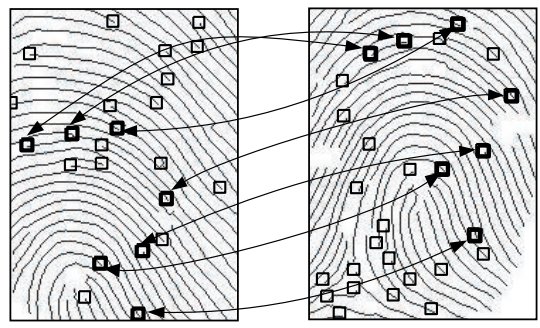

(c)

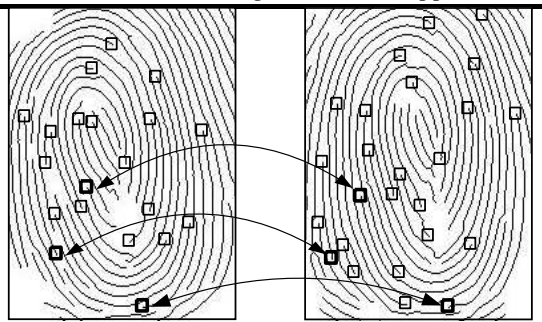

(b)

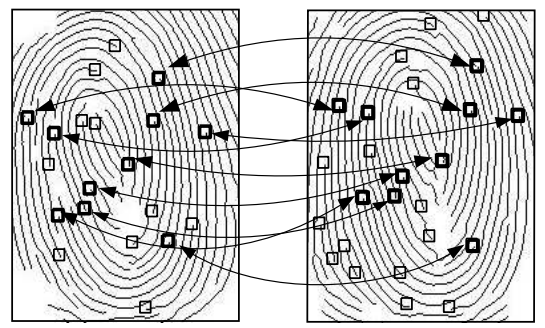

(d)

Figure 2: Improvement in correspondences (indicated by arrows) between two sets of minutiae point patterns. (a) and (b) are correspondences obtained by the EPPM algorithm while (c) and (d) are correspondences obtained by the new algorithm for the same pairs of fi ngerprint images.

1. Given a pair of images, $I_{0}$ (called template) and $I_{1}$ (called query), we extract minutiae points using the method proposed in [9]. Assume $M_{0}=$ $\left(u_{1}, u_{2}, \ldots, u_{m_{0}}\right)$ is the set of minutiae points extracted from $I_{0}$ and $M_{1}=\left(v_{1}, v_{2}, \ldots, v_{m_{1}}\right)$ from $I_{1}$. We also trace the ridge associated with each minutiae point and extract sample points on that ridge (sampled every 5-th point), with $R_{u_{i}}=\left(u_{i, 1}^{*}, u_{i, 2}^{*}, \ldots, u_{i, a}^{*}\right)$ and $R_{v_{j}}=\left(v_{j, 1}^{*}, v_{j, 2}^{*}, \ldots, v_{j, b}^{*}\right)$ denoting the ridge points corresponding to the minutiae $u_{i}$ and $v_{j}$, respectively.

2. For a total of $m_{0} \cdot m_{1}$ possible pairings of minutiae points, we select one $\left(u_{i}, v_{j}\right),\left(i=1,2, \ldots, m_{0}\right.$, $\left.j=1,2, \ldots, m_{1}\right)$ as the reference pair and transform remaining minutiae points in $M_{0}$ and $M_{1}$,respectively, to polar coordinates with centers at $u_{i}$ and $v_{j}$.

3. The query minutiae set $M_{1}$ is rotated about its reference minutiae $v_{j}$ in order to find candidate pairings with $M_{0}$. Each candidate pair is verified using a bounding box. Let $M P_{i j}$ be the total number of matching pairs for $\left(u_{i}, v_{j}\right)$.

4. Compute $\left(\Delta x_{i j}, \Delta y_{i j}, \Delta \theta_{i j}\right)$ as the translational (in $\mathrm{x}$ and y directions) and rotational offsets associated with $\left(u_{i}, v_{j}\right)$. Then add votes to $\operatorname{Vote}\left(\Delta x_{i j}, \Delta y_{i j}, \Delta \theta_{i j}\right)$ with the number of votes equal to $M P_{i j}$.

5. Perform steps $2-4$ until all possible reference pairs are considered and votes for the corresponding transformation parameters are obtained.
6. Establish bins with size of $(20,20,15)$ and collect votes for each bin. The top 5 bins with the maximum number of votes are selected and each $\left(\Delta x_{i j}, \Delta y_{i j}, \Delta \theta_{i j}\right)$ that contribute to one of the top 5 bins are sent to a 2-D dynamic matching algorithm to get the matching score [13].

7. The one that results in the highest matching score is finally chosen as the optimal global transformation and is used to establish the individual minutiae correspondences within a bounding box.

Fig. 2 shows examples of correspondences generated by using the original EPPM algorithm [9] and the modified algorithm. Let $(T / C)$ denote the ratio of the detected correspondences to the number of correct correspondences that are manually verified. Figs. 2(c) and 2(d) give higher ratios of $(7 / 7)$ and (10/10) compared to Figs. 2(a) and 2(b) that give low values of $(0 / 4)$ and $(1 / 3)$.

\section{Incorporating Minutiae Orientation}

One difficulty in incorporating minutiae orientation information is that transformation in the orientation space is not equivalent to transformation in the the spatial domain; for example, rotation in the spatial domain corresponds to translation in the orientation space. Thus, in order to use the TPS to model deformations using minutiae locations and orientations, we convert the orientation information into the spatial domain using the following method. 


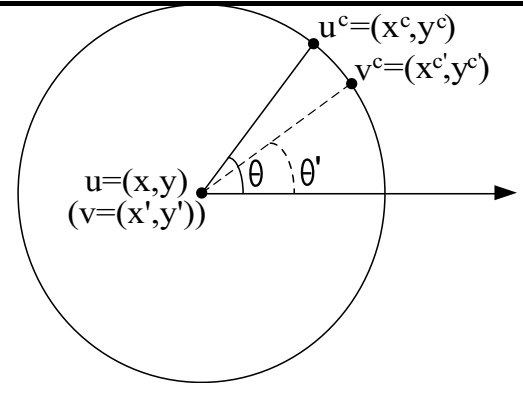

Figure 3: Obtaining angle points $u^{c}$ and $v^{c}$ based on the minutiae orientations $\theta$ and $\theta^{\prime}$. Note that $u$ and $v$ are aligned for simplicity of illustration.

Let the location and orientation of a minutiae point be denoted by $u=(x, y)$ and $\theta$, respectively. We draw a unit circle around $u$ and find its corresponding "angle point" $u^{c}=\left(x^{c}, y^{c}\right)$, where:

$$
x^{c}=x+\cos \theta, y^{c}=y+\sin \theta .
$$

Assume $u$ is in correspondence with the minutiae point $v=\left(x^{\prime}, y^{\prime}\right)$. The angle point $v^{c}=\left(x^{c}, y^{c}\right)$ of $v$ is found in the same way using the minutiae orientation $\theta^{\prime}$ for $v$ (see Fig. 3). Therefore, orientation information is converted into the spatial domain as we generate an angle point pair for each corresponding minutiae pair. As a result, the number of corresponding point patterns for the TPS model is doubled. The TPS deformation model is found as in Section 2 with $U$ and $V$ now containing both minutiae points and angle points.

\section{Average Deformation Models}

Suppose we have $N$ impressions of a finger, $I_{1}, I_{2}, \ldots, I_{N}$. Each impression $I_{k}$ can be paired with the remaining impressions to create $N-1$ pairs of the form $\left(I_{i}, I_{j}\right), i \neq j$. For the pair $\left(I_{i}, I_{j}\right)$, we obtain a non-linear deformation model $F_{i j}$ by employing the TPS technique described in Section 2 . Note that $F_{i j}$ then transforms every pixel in the template fingerprint $I_{i}$, to a new location. Thus, we can compute the average deformation of each pixel $u$ in $I_{i}$ as:

$$
\bar{F}_{i}(u)=\frac{1}{N-1} \sum_{\substack{j=1 \\ j \neq i}}^{N} F_{i j}(u) .
$$

The average deformation is the typical deformation that arises when we compare one impression of a finger (the baseline impression) with others of the same finger. This offline process is repeated for every impression $I_{i}, i=$ $1,2, . ., N$, resulting in $N$ average deformation models that can be incorporated in the matching stage.

\subsection{Index of Deformation}

In $[1,2]$, a method to rank the average deformation models using the pixel-wise covariance matrix of the deformations was given. However, this method is computationally expensive and does not necessarily reflect non-linear deformations because of its sensitivity to linear transformations. We propose a new ranking method based on the bending energy, a measure of non-linear deformations that the TPS tries to minimize. The minimized bending energy can be computed by

$$
J(F)=\operatorname{trace}\left(W^{T} S W\right),
$$

where $W$ is the parameter for the non-linear transformation and $S$ is the distance matrix (see equation (4)).

Given $N$ impressions per finger, if we choose $I_{i}$ as the template image, $N-1$ deformation functions $F_{i j}$ will be obtained when $I_{i}$ is compared to $I_{j}, j=1,2, \ldots, N, j \neq i$. Thus, a total of $N-1$ values of bending energy $J(F)$, for $F=F_{i j}, j=1,2, \ldots, N, j \neq i$, are provided. The $\Phi$-index of deformation for template $I_{i}$ is then defined as:

$$
\begin{gathered}
\Phi_{i}=\frac{1}{N-1} \sum_{\substack{j=1 \\
j \neq i}}^{N}\left(J\left(F_{i j}\right)-\bar{J}_{i}\right)^{2}, \\
\text { where } \quad \bar{J}_{i}=\frac{1}{N-1} \sum_{\substack{j=1 \\
j \neq i}}^{N} J\left(F_{i j}\right) .
\end{gathered}
$$

Low (high) values of the $\Phi$-index indicate that the amount of bending energy applied by each deformation function is similar (dissimilar) to each other. However, low values of the $\Phi$-index also occurs when the number of correspondences is consistently small between a template and other impressions of the same finger, which should not be considered as a potentially good template. Therefore, for each template $I_{i}$, we compute its total number of correspondences $C_{i}$ with each $I_{j}, j=1,2, \ldots, N, j \neq i$ and the mean of total correspondences for impressions of a finger by $\bar{C}=\frac{1}{N} \sum C_{i}, i=1,2, \ldots, N$. We exclude those templates with $C_{i}<\bar{C}, i=1,2, \ldots, N$, during the ranking. Among the remaining templates, the one $I_{i^{*}}$ with $i^{*}=\operatorname{argmin}_{i: C_{i} \geq \bar{C}} \Phi_{i}$, is the optimal template for a finger. It is considered to have the smallest variability in the nonlinear deformations compared to all other templates (Fig.4).

\section{Experimental Results}

In order to apply the TPS model to reliably estimate fingerprint deformation, we need to have several impressions of the same finger (at least 10). Large number of impressions of a finger are not available in standard fingerprint 


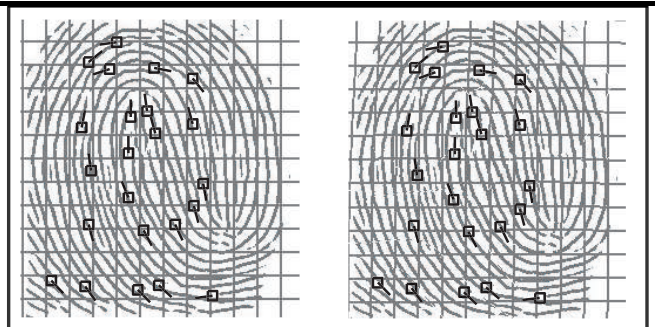

(a) $\Phi=0.0030$

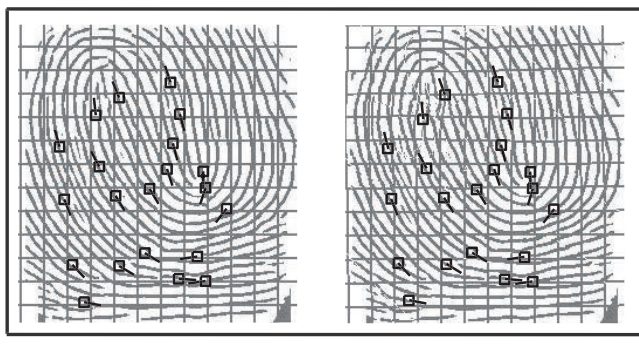

(c) $\Phi=0.0242$

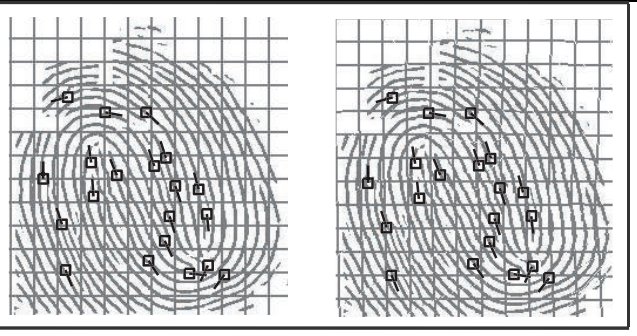

(b) $\Phi=0.0151$

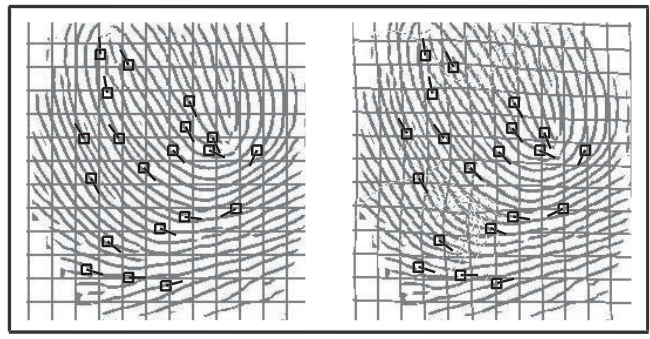

(d) $\Phi=0.1061$

Figure 4: Four templates before (left) and after (right) applying the average deformation model based on minutiae locations and orientations are shown on a reference grid with minutiaes marked. They correspond to (a) the highest $\Phi$-value (b) the 6 -th highest $\Phi$-value (c) the 11 -th highest $\Phi$-value and (d) the lowest $\Phi$-value when ranked using the bending energy.

databases (e.g., FVC 2002 [11]) and therefore, we used data collected in our lab. We acquired fingerprint images of 50 fingers using the Identix Bio Touch USB 200 optical sensor $(256 \times 255,380 \mathrm{dpi})$ over a period of two weeks. There were 32 impressions corresponding to every finger, resulting in a total of 1600 impressions. One half of the impressions (16 impressions per finger, with a total of 800 impressions) were used as template to compute the deformation model while the remaining 800 impressions were used as query images for testing. The minutiae points and their corresponding orientation information were extracted using the algorithm outlined in [9]. For the 16 test impressions per finger, the deformations $F_{i j}$ based on minutiae locations and orientations were obtained by fixing $I_{i}$ as the template and the remaining $I_{j}, j \neq i$ as the query with $\lambda=5$ in equation (5). The average deformation model $F_{i}$ was then computed using equation (8). During the matching stage, $M_{i}$, the minutiae set of $I_{i}$, was deformed to $M D_{i}=\bar{F}_{i}\left(M_{i}\right)$. Thus, a total of 800 sets $(50 \times 16)$ of average deformation models were obtained. We also use $M D_{i}^{*}$ to denote the deformed minutiae set using the TPS model based only on minutiae locations [1].

In the first experiment, every template image $I_{i}$ was compared with every query image $I_{j}$. A modified matcher based on the 2-D dynamic matching algorithm in [13] was used to generate three types of matching scores for each comparison: the matching score obtained by matching (i) $M_{i}$ with $M_{j}$ (no adjustment for deformations), (ii) $M D_{i}^{*}$ with $M_{j}$ (deformed based on minutiae locations only) and (iii)
$M D_{i}$ with $M_{j}$ (deformed based on minutiae locations and orientations). The Receiver Operating characteristic (ROC) curve plotting the genuine accept rate (GAR) against the false accept rate (FAR) at various thresholds is presented in Fig. 5(a). When the average deformation model based on minutiae locations is applied to pre-distort the template prior to matching, an improvement of approximately $3.0 \%$ with respect to the non-deformed templates is observed at a false accept rate of $0.01 \%$. Using minutiae locations and orientations results in an additional $\approx 1.0 \%$ improvement. In the second experiment, the advantage of using the $\Phi$ index is demonstrated. We generate two sets of optimal templates using (a) the knowledge of average pixel-wise deformation and (b) the knowledge of the bending energy. Each set contains 50 optimal templates (one per finger) from the training data and 800 queries from the test data. Fig. 5(b) shows the ROC curve by applying these two optimal sets, together with the ROC curve using method (i) in the first experiment. We can see that the ranking method based on the bending energy not only provides computational efficiency, but also leads to better performance in matching.

\section{Summary and Conclusions}

We have proposed a method to generate average deformation models based on minutiae locations and orientations. By utilizing minutiae orientations, the proposed method improves the performance of a fingerprint matching system. A modified correspondence algorithm is also proposed to gen- 


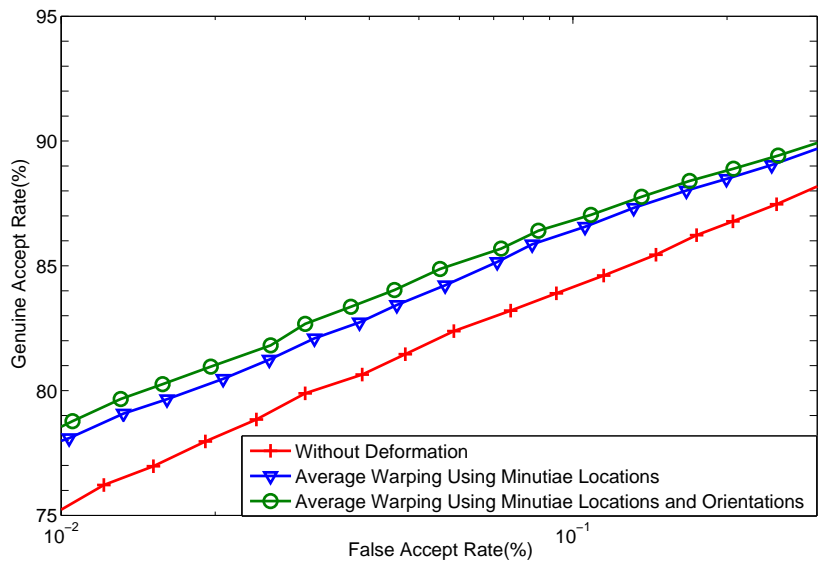

(a)

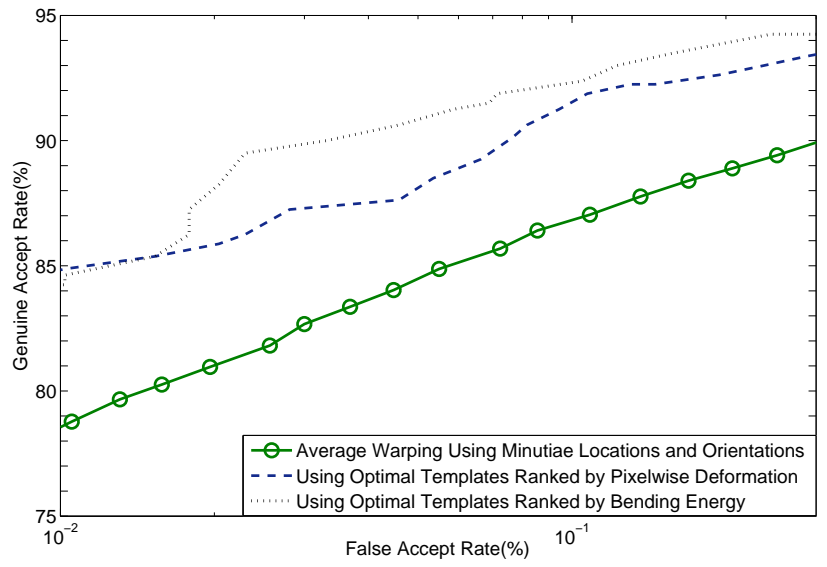

(b)

Figure 5: (a) Improvement in matching performance using non-deformed templates (' $\times$ '), deformed templates using the average deformation model based on: minutiae locations (' $\nabla$ ') and minutiae locations and orientations (' $\circ$ '). (b) Improvement in matching performance using all deformed templates (' $\circ$ '), the optimal templates ranked by $\Phi$-index based on: pixel-wise covariance of deformation ('- -') and bending energy ('...') when the average deformation model based on minutiae locations and orientations is applied.

erate reliable correspondences prior to computing the TPS model. In addition, a more efficient and robust measure to generate the index of deformation is defined by using the bending energy of deformations. Future work includes incorporating more features like ridges and ridge orientations into the framework. We also plan to adopt an incremental approach to update the average deformation model in a coarse-to-fine fashion.

\section{References}

[1] A. Ross, S.C. Dass and A.K. Jain, "A Deformable Model for Fingerprint Matching," Pattern Recognition, Vol.38(1), pp. 95-103, 2005.

[2] A. Ross, S.C. Dass and A.K. Jain, 'Estimating Fingerprint Deformation," Proceedings of the International Conference on Biometric Authentication (ICBA), Vol. 3072, pp. 249255, Hong Kong, 2004.

[3] N.K. Ratha and R.M. Bolle, 'Effect of controlled image acquisition on fi ngerprint matching," Proceedings of the 14th IAPR International Conference on Pattern Recognition, Vol. 2, pp. 1659-1661, Brisbane, 1998.

[4] A. Senior and R. Bolle, 'Improved fi ngerprint matching by distortion removal,' IEICE Transactions on Information and System, Special issue on Biometrics, Vol. E84-D(7), pp. 825831, 2001.

[5] A.M. Bazen and S.H. Gerez, "Elastic minutiae matching by means of thin-plate spline models," Proceedings of 16th ICPR International Conference on Pattern Recognition, Québec City, Canada, 2002.
[6] A.M. Bazen and S.H. Gerez, 'Fingerprint matching by thin-plate spline modeling of elastic deformations," Pattern Recognition, Vol. 36(8), pp. 1859-1867, 2003.

[7] Z.M. Kovács-Vajna, "A fi ngerprint verifi cation system based on triangular matching and dynamic time warping," IEEE Transactions on Pattern Analysis and Machine Intelligence, Vol. 22(11), pp. 1266-1276, 2000.

[8] F.L. Bookstein, 'Principal warps: Thin plate splines and the decomposition of deformations," IEEE Transactions on Pattern Analysis and Machine Intelligence, Vol. 11(6), pp. 567585.

[9] A.K. Jain, L. Hong and R. Bolle, 'On-line fi ngerprint verifi cation,' IEEE Trasactions on Pattern Analysis and Machine Intelligence, Vol. 19(4), pp. 302-314, 1997.

[10] I.L. Dryden and K.V. Mardia, "Statistical Shape Analysis," John Wiley and Sons, 1998.

[11] D. Maio, D. Maltoni and R. Cappelli and J. L. Wayman and A. K. Jain, 'Fingerprint verifi cation competition,' Proceedings of the 16th International Conference on Pattern Recognition (ICPR), Québec City, Canada, 2002.

[12] C. Watson, P. Grother and D. Cassasent, 'Distortion-tolerant fi lter for elastic distorted fi ngerprint matching," Proceedings of SPIE Optical Pattern Recognition, pp. 166-174, 2000.

[13] A.K. Jain, S.Prabhakar and S.Chen, 'Combining Multiple Matchers for a High Security Fingerprint Verifi cation System," Pattern Recognition Letters, Vol 20, No. 11-13, pp. 1371-1379, 1999. 